\section{CONDICIONES, MEDIOAMBIENTE Y RIESGOS PSICOSOCIALES DEL TRABAJO DOCENTE EN CORRIENTES}

\author{
CONDITIONS, ENVIRONMENT AND PSYCHOSOCIAL RISKS \\ OF TEACHING WORK IN CORRIENTES
}

\author{
Mirian Graciela Arce
}

\section{RESUMEN}

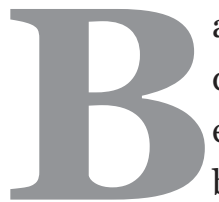

asado en la creciente importancia que tomaron en las últimas décadas los estudios sobre factores de Riesgos Psicosociales en el Trabajo y de la incorporación de visiones más actualizadas sobre Condiciones y Medioambiente de Trabajo, con este artículo se propone indagar la situación de tales cuestiones en la realidad Argentina a nivel subnacional, focalizando en los docentes de Corrientes. La disquisición se cimenta en el análisis de las encuestas aplicadas al sector, que se realizaron dentro del Proyecto de Investigación sobre Riesgos Psicosociales en el Trabajo que se desarrolla en la Facultad de Ciencias Económicas de la UNNE. El principal objetivo consiste en realizar una interpretación conjugando los datos provenientes de las entrevistas con las posiciones teóricas sobre el tema, a los efectos de describir la manera en que se manifiesta el fenómeno en el sector.

Palabras clave: educación, factores de riesgo, ambiente laboral.

\section{ABSTRACT}

Based on the importance of the factors of Psychosocial Risks at Work and updated views on Work Conditions and Environment, this paper investigates the situation in Argentina at the subnational level, focusing on the teaching sector of Corrientes. The research is based on the analysis of the interviews with teachers, carried out in the Research Project on Psychosocial Risks at Work that is developed in the Faculty of Economic Sciences of the UNNE. The objective is to interpret the interview data with theories on the subject, to describe how the phenomenon manifests itself in this group.

Keywords: education, risk factors, work environment.
Mirian Graciela Arce miriangracielaarce@yahoo.com.ar

Facultad de Ciencias Económicas

Universidad Nacional del Nordeste ARGENTINA

\section{COMO CITAR ESTE ARTÍCULO} Arce, M. G. (2019). Juventudes y Condiciones, medioambiente y riesgos psicosociales del trabajo docente en Corrientes. Revista de la Facultad de Ciencias Económicas, 23( 2), 91 - 107. http://dx.doi.org/10.30972/rfce.2324021

\section{()요 $\Theta \Theta \Theta$}

https://creativecommons.org/licenses/by-nc-nd/4.o/

Revista de la Facultad de Ciencias Económicas ISSN 1668-6357 (formato impreso) ISSN 1668-6365 (formato digital) por Facultad de Ciencias Económicas Universidad Nacional del Nordeste (UNNE) Argentina se distribuye bajo una Licencia Creative Commons Atribución - No Comercial - Sin Obra Derivada 4.o Internacional. 


\section{INTRODUCCIÓN}

Este artículo se enmarca dentro del PI 18Mo07/19: "Riesgos psicosociales en el trabajo. Percepción de los trabajadores e impacto en las empresas y organizaciones" -Facultad de Ciencias Económicas, UNNE-. El mismo se sitúa dentro del enfoque de las Condiciones y Medioambiente de trabajo (CyMAT) y de los Riesgos Psicosociales del trabajo (RPST), que en la actualidad forman parte importante de los estudios interdisciplinarios sobre el mundo del Trabajo.

Desde la concepción que aquí se adopta, se entiende que las CyMAT se articulan con los RPST, ocasionando daños sobre la salud física, emocional y psíquica del trabajador. Las CyMAT "están constituidas por los factores socio-técnicos y organizacionales del proceso de producción implantado en el establecimiento (o condiciones de trabajo) y por los factores de riesgo del medio ambiente de trabajo" (Neffa, 2018, p. 37). Los RPST refieren a los elementos presentes en la relación laboral como las condiciones del empleo, de la organización, el contenido del proceso del trabajo y las relaciones sociales dentro de él, que pueden perturbar las dimensiones cognitivas y psíquicas del trabajador. Son "los aspectos del diseño y la gestión del proceso de trabajo y sus contextos sociales y organizativos que cuando son dañinos pueden llegar a causar daño psicológico o físico" (Neffa, 2018, p.50).

La investigación es cualitativa mixta. El problema objeto de estudio son las CyMAT y los RPST a nivel subnacional, delimitado espacialmente en la Provincia de Corrientes, Argentina. La principal técnica metodológica consiste en la observación de datos y documentos aplicada al análisis de las encuestas realizadas en 2014 a los docentes de nivel primario y a la bibliografía académica sobre el tema. El objetivo que se persigue busca analizar aquellos datos a la luz de las construcciones teóricas. Para lo cual se sintetizan las concepciones tradicional y renovadora de las CyMAT y los modelos de Karasek-Johnson-Theorell, J. Siegrist y los factores de Riesgo propuestos por Gollac, tomando como fuente los estudios del Dr. Julio César Neffa. Esto con el fin de describir cómo se manifiesta el fenómeno en los trabajadores de la docencia en Corrientes.

Para presentar la información se organizaron cinco categorías mediante las cuales se pretende englobar las posiciones teóricas que constituyen el marco de referencia. Estas son: demanda psicológica y esfuerzo requerido; autonomía y margen de control sobre la actividad; recompensa; CyMAT; e inseguridad en el trabajo.

\section{ANTECEDENTES Y MARCO TEÓRICO}

Las CyMAT se distinguen en las concepciones Tradicional y Renovadora (Neffa, 2018). Para la Concepción Tradicional, la salud de los trabajadores se reduce a la ausencia de 
enfermedad sin advertir que las CyMAT son causales de ésta, pues someten al trabajador en su vida laboral a un deterioro progresivo de su salud. La misma se enfoca en la Seguridad e Higiene en el trabajo y sólo luego en la medicina laboral, por ello cuando su perspectiva invade la esfera del Estado, provoca un sistema de regulaciones dirigidas a la protección individual, obligando a cumplir determinadas leyes y medidas de seguridad y a la reparación del daño en caso de accidentes de trabajo. Sin embargo, no considera todos los factores de riesgo de las CyMAT, ni la 'prevención' como forma de mejorar tales condiciones. Al situarse en el nivel microeconómico, sin considerar su contexto socio-económico y los condicionamientos históricos, no logra identificar causas profundas, lo que impide la articulación con políticas de prevención globales e integradas que vayan más allá de la situación presente (Neffa, 2018).

Como reduce al trabajador a su dimensión física, solo observa el cuerpo y sus aspectos biológicos, eludiendo las facetas relacionales, afectivas, cognitivas y los elementos que conforman su carga psíquica. Su análisis individualizado y parcializado plantea la existencia de un 'trabajador promedio', constructo ideal a través del cual se miden tiempo, movimientos y en este caso, los máximos admisibles de exposición a Riesgos. Dentro de esta postura el trabajo se interpreta como castigo u obligación, naturalizando el dolor y fatiga que provoca, con lo cual vuelve los riesgos inherentes al trabajo mismo. Por ende, como el riesgo no se puede eliminar, se pone un precio al deterioro de la salud, los accidentes y enfermedades que sufre el trabajador que lo realiza, mercantilizando la salud junto con el trabajo, la que se incluye dentro del paquete que se vende a la patronal y en tanto bien comercializable está sujeto a un precio de mercado.

Esta concepción recoge su herencia del mecanicismo y el fatalismo inspirados en el positivismo del siglo XIX. Así, identifica los factores de riesgo y busca solucionarlos según la relación causa-efecto, donde la responsabilidad mayor recae sobre el trabajador. Otra de sus fuentes es el liberalismo e individualismo del siglo XIX, lo que lleva a desconocer la existencia de un colectivo de trabajo -que no es igual a la suma de individuos aislados- que es el que está expuesto en su conjunto al riesgo (Neffa, 2018). Dentro de esta trama el debate sobre los Riesgos no incorpora al trabajador, perdiendo una oportunidad valiosa para identificarlos. Asimismo, al no trabajar interdisciplinarmente, crea escuelas aisladas que no logran una conexión entre sí, por lo que el tema se aborda desde cada especificidad y no de forma integral.

Para la Concepción Renovadora trabajo y salud están intrínsecamente relacionados. La salud no implica solamente ausencia de enfermedad sino bienestar en general, con lo cual adosa la idea de prevención. Este enfoque integra los aspectos objetivos de la concepción anterior con la vertiente subjetiva (saberes no codificados), por lo que involucra a los trabajadores en los procesos de análisis. También sostiene que las causas de los riesgos son múltiples y complejas, por ello deja de lado la visión simplista causa-efecto donde el accidente suele atribuirse al mal desempeño del factor humano, para reflexionar retrospectivamente intentando visualizar la concatenación de factores causales (Neffa, 2018). Como trabajo y riesgo no son sinó- 
nimos, pues el trabajo no es necesariamente riesgoso, los elementos que lo provocan pueden ser eliminados. En este sentido el trabajador accidentado es una víctima en un entorno donde la patronal es la responsable de brindar seguridad. Así, la salud no es una mercancía y que el trabajo sea benéfico para las personas depende de las CyMAT, que al estar condicionadas por los procesos de trabajo son dinámicas y cambiantes.

Al contemplar a los seres humanos como heterogéneos, pues los integrantes de un colectivo de trabajo pueden diferenciarse por edad, sexo, origen profesional, medio geográfico e histórico en el que nacieron y crecieron, personalidad, capacidades físicas, hábitos, entre otros factores, rechaza la idea de 'trabajador promedio', a la que estima la excepción y no la regla. En este contexto, el trabajo no es visto como un castigo sino como un derecho y una obligación social tendiente a la perpetuación de la especie y al progreso científico y tecnológico que repercute en el mejoramiento general de las condiciones de vida.

Su visión global, integrada y multidisciplinaria se fundamenta en la teoría de sistemas para reafirmar que las CyMAT no pueden estudiarse de forma aislada e individual. Principalmente por la sinergia entre los factores cuando se combinan, los cuales provocan que el proceso de trabajo no afecte sólo a una parte del trabajador sino a toda su persona, incluyendo los aspectos físicos, psíquicos, cognitivos, afectivos y en tanto el trabajador se halla inserto dentro de un colectivo, también a las relaciones sociales (Neffa, 2018).

Sobre los RPST, Neffa (2015) sostiene que los modelos de Karasek-Johnson-Theorell, confrontan las demandas psicosociales de la organización con la autonomía y margen de control del trabajador, el de J. Siegrist mide el equilibrio o desequilibrio entre la reciprocidadrecompensa con la intensidad del trabajo y el de Gollac identifica seis factores de RPST.

Robert Karasek (citado por Neffa, 2015) formuló el modelo demanda-autonomía-control, mediante el cual explica el estrés laboral en función del balance entre dos partes: las demandas organizacionales y emocionales-psicológicas del trabajo, el nivel de control que tiene el trabajador sobre éste y su grado de autonomía (latitud decisional) para adecuar su actividad al trabajo prescripto. En este contexto, una fuerte demanda de trabajo con una autonomía reducida se relaciona con posibles perturbaciones psiquiátricas. Cuando el trabajo exige comportamientos sociales complejos y planificados que generan emociones, aumentan los riesgos de perturbación del humor. También estima que las perturbaciones depresivas están muy relacionadas con la pérdida de control del individuo sobre sí mismo y su entorno. Este control, está asociado a las oportunidades de desarrollar habilidades propias y tener cierta autonomía en su ejecución, que dependen del nivel de educación, formación profesional, experiencia en el puesto de trabajo y conocimiento tácito de los que dispone cada trabajador. No obstante, la apropiación de estas competencias, conducen a que el trabajador se resista a los trabajos repetitivos y monótonos, aunque ofrece la oportunidad de crear trabajadores polivalentes. Así, una demanda de trabajo asociada con tareas complejas puede generar perturbaciones de humor y riesgos de ansiedad, pero también pueden hacerlo las tareas prescriptas, dado que el traba- 
jador requiere violar el manual de procedimientos para realizarlas, en tanto es poco factible que lo plasmado en el papel ocurra exactamente en la realidad. A pesar de ello, para Karasek (citado por Neffa, 2015) el estrés no depende tanto de la cantidad de demandas como de la falta de autonomía. Incluso, remarca la importancia de la influencia del trabajador en su puesto, entendida como el margen de decisión y autonomía del trabajador respecto al contenido y condiciones de su trabajo, por lo que una baja influencia aumenta el riesgo de enfermedades. Por ello, que el trabajador goce de autonomía para controlar su trabajo es visto desde esta perspectiva como fuente de desarrollo personal y factor positivo para preservar la salud. Por el contrario, la heteronomía que genera la división social y técnica del trabajo, donde el trabajador está subordinado al trabajo prescripto, es vista como factor de riesgo y dependiendo de la exposición a tales condiciones de trabajo generará un mayor o menor impacto negativo sobre la salud.

Karasek (citado por Neffa, 2015) parte de una concepción cuantitativa respecto a las exigencias que el proceso de trabajo genera sobre las dimensiones psíquicas o mentales del trabajador. Por ello intenta medir cuánto es el volumen de trabajo con relación al tiempo disponible para hacerlo, cuya presión se intensifica cuando el tiempo de realización es más acotado. Siguiendo lo que postula este modelo, se identifican cuatro situaciones relacionadas con las demandas psicosociales del trabajo en relación con el margen de autonomía y maniobra de la que dispone el trabajador, que tienen consecuencias sobre su salud. Si la intensidad es fuerte pero hay mucha autonomía el trabajo estimula el involucramiento. Si la intensidad es débil pero la autonomía es alta, el trabajo es más tranquilo y saludable. Si la intensidad es fuerte y no hay mucha autonomía, la carga del trabajo genera fuertes tensiones provocando efectos negativos sobre la salud. Si la intensidad es débil y no hay mucha autonomía, el trabajo se vuelve pasivo y rutinario, estimulando la pérdida de interés en la tarea. La principal hipótesis del modelo postula que los niveles más bajos de bienestar psicológico y los niveles más altos de síntomas y enfermedades se encuentran en el punto tres.

Neffa (2015) considera que quienes aportaron al modelo de Karasek fueron Johnson y Hall y luego Theorell, proporcionando la dimensión del apoyo social. La función de éste estriba en "incrementar la capacidad de un trabajador para hacer frente a una situación estresante" (Neffa, 2015, p.260). Lo que resulta en que la tensión del trabajo pueda moderarse en una situación de trabajo con alto apoyo, dado que aquel funciona como un amortiguador del efecto del stress en la salud y se relaciona con el clima de trabajo. El apoyo social tiene dos componentes: el social-horizontal, donde el trabajador recibe apoyo social y emocional de sus colegas, que permite transferir conocimientos y experiencias, enseñar las reglas del oficio, darse ánimo en momentos afectivos difíciles, lo que crean lazos de solidaridad, comunicación y cooperación (Neffa, 2015, p. 261) y el apoyo técnico e instrumental, que adopta las modalidades: formación 
adecuada para ejecutar la tarea y/o asesoramiento técnico-profesional, proporcionado por la jerarquía de la organización (Neffa, 2015, pp. 260-261). El apoyo social admite cuatro modalidades: orientación directiva; ayuda no directiva; interacción social positiva y ayuda tangible. De modo que, un apoyo social débil presenta riesgos para la salud del trabajador porque lo hace experimentar alta tensión, pero el riesgo asociado a la tensión del trabajo disminuye si se cuenta con alto nivel de apoyo.

Siegrist (citado por Neffa, 2015) confronta el desequilibrio entre el esfuerzo o exigencia que demanda la organización con la recompensa del trabajo, proponiendo el modelo esfuerzorecompensa. Así, "La intensidad de la demanda al trabajador se define de manera simplificada como la relación entre la cantidad de trabajo y el tiempo disponible para realizarlo: volumen, ritmo, interrupciones e intensidad de trabajo" (Neffa, 2015, p.268). Se estima que una demanda alta de trabajo se produce cuando se asigna al trabajador más trabajo del que puede realizar en un tiempo preestablecido. Esta intensidad no sólo se relaciona con la mala planificación del trabajo o ineficiencias en sus procesos, sino con cuestiones salariales. Por ello, cuando el esfuerzo invertido por el trabajador es más elevado que la recompensa, se produce un efecto negativo sobre la salud del mismo. Esto requiere equilibrar demandas y recompensas. Como cita Neffa (2015) "Existe evidencia científica de que la exposición crónica a una situación de alta intensidad sumada a bajas recompensas contribuye a incrementar de manera significativa el estrés y provoca desórdenes mentales y físicos" (p.268). Cabe destacar que "las exigencias (físicas, psíquicas, mentales) pueden ser intrínsecas o extrínsecas al sujeto; las recompensas pueden ser materiales, morales o simbólicas” (Neffa, 2015, p. 272). La amenaza de despido; el desempleo; la precariedad laboral; los cambios en las condiciones de trabajo; la degradación de la categoría; la falta de expectativas de crecimiento; los obstáculos para el desarrollo personal; la inconsistencia de status; la flexibilización del mercado de trabajo; la aparición de los empleos atípicos y también la expansión del sector terciario y de servicios, son variables a considerar en este modelo porque incrementan la vulnerabilidad del trabajador mientras que lo someten a un trabajo intensivo con baja recompensa. Siguiendo a Neffa (2015) se puede sintetizar este modelo en los siguientes puntos. Fuerte exigencia con adecuada recompensa, fatiga pero provoca involucramiento y satisfacción. Fuerte exigencia y recompensa insuficiente, provocan tensión, desagrado, insatisfacción, que conducen a la rotación o abandono del puesto. Débil exigencia y adecuada recompensa, generan un trabajo rutinario que disminuye la motivación. Débil exigencia y recompensa inadecuada provocan poco involucramiento, cambio de trabajo, ausentismo, etc.

Neffa (2015) considera que los mencionados modelos de RPST, son complementarios pues ofrecen tres variables desde las cuales abordar estudios que permitan detecta el impacto sobre la salud psíquica y mental del trabajador (Neffa, 2015, pp. 278-279).

Para Gollac (citado por Neffa, 2015) los RPST son aquellos riesgos para la salud mental, física y social, provocados por las condiciones de empleo y los factores organizacionales y relacionales 
que son susceptibles de interactuar con el funcionamiento mental. El esquema que propone ilustra la dinámica sufrimiento-salud-enfermedad, que se origina en el proceso de trabajo a causa del contenido y organización del mismo. En este se sostiene que, si desde el inicio el trabajador sublima el sufrimiento (transforma el impulso en un acto más aceptable social o moralmente), los impactos sobre la salud son menos dañinos, de no ser así, los trabajadores construyen estrategias colectivas y si éstas también fracasan el sufrimiento predispone a enfermedades.

Los factores de RPST según Gollac (citado por Neffa, 2015), se organizan en función de los siguientes ejes:

La demanda psicológica y el esfuerzo requerido. Habla de la cantidad, ritmo e intensidad del trabajo, que determina la carga psíquica, cognitiva, mental y global del mismo.

El stress. Aparece cuando la adaptación y resistencia a los riesgos y amenazas del proceso de trabajo no se logra, y las demandas externas (sociales u organizacionales) o internas (psicológicas o cognoscitivas) superan la capacidad de respuesta del individuo, produciendo desequilibrios que propenden a enfermedades.

Las exigencias emocionales. Surgen cuando el trabajador se encuentra en la obligación de esconder o negar sus emociones por diversas razones, entre ellas, las exigencias del puesto de quienes están frente al área de reclamos o atienden al público y deben estar siempre sonrientes.

La autonomía en el trabajo. Implica la intervención activa en el proceso de trabajo, utilizando las competencias profesionales y decidiendo sobre la producción de bienes y servicios, donde se incluye el autodireccionar la propia carrera y vida profesional.

Las relaciones sociales en el trabajo. Refieren a las vinculaciones con compañeros, jefes, demás miembros de la organización e interacciones con clientes, usuarios, pacientes y/o consumidores, que son los responsables del clima en el lugar de trabajo. Si estas relaciones son inadecuadas para generar un clima aceptable, si no hay estímulos, predisposición al involucramiento, ambiente de integración y cooperación, o hay falta de reconocimiento, injusticias, reducido apoyo social y técnico, el trabajador puede experimentar situaciones estresantes como discriminación, hostigamiento, violencia o acoso.

Conflictos éticos y/o de valores. Suceden cuando el trabajador está obligado a confrontar su sentido del trabajo con las condiciones en que lo realiza; cuando choca con aspectos morales donde la tarea obliga a cotejar permanente los valores de la persona con los de la organización; o cuando el trabajador siente que el trabajo que realiza es inútil o no contribuye en nada, lo que conduce a que subestime su actividad.

La inseguridad en la situación de trabajo y empleo. Asociada al dominio del trabajador sobre su destino presente y futuro, la planificación de su carrera y el manejo de la incertidumbre. Ataña a los empleos precarios con riesgo de despido, reducción o suspensión del salario y a situaciones de obstaculización de ascensos o de carrera; desaparición de empleos de planta permanente que generan incertidumbre sobre la jubilación; introducción de nueva tecnología 
que predispone al temor de perder el puesto si no se logra manejarla; y a las nuevas formas de organización del trabajo, que requieren otras competencias como el trabajo en equipo.

\section{ANÁLISIS DE LOS DATOS}

A los efectos de encuadrar el análisis en las características socioeconómicas de los encuestados, cabe señalar que el 99\% de los docentes pertenece al sector público. La mayoría trabaja en organizaciones con mucho personal y reviste como titular o interino, que encuadra como trabajo de planta permanente. El 82\% realiza tareas de enseñanza, mientras el resto ocupa cargos directivos, de coordinación, administrativos y técnicos. El $79 \%$ son mujeres, el 21\% varones y sus principales edades oscilan entre los 35 a 44 años y 45 a 54 años. En la condición de jefe/a de hogar, el $57 \%$ es responsable total del sustento familiar, mientras el $36 \%$ colabora con éste.

\subsection{Demanda psicológica y esfuerzo requerido}

Para Gollac (citado por Neffa, 2015) las demandas psicológicas y el esfuerzo requerido se relacionan directamente con los tiempos, ritmos e intensidad del trabajo. La interferencia sobre alguno de estos factores modifica la carga psíquica, mental y general aplicada al mismo potenciando los riesgos. Ahora bien, las personas no siempre son conscientes del tiempo que insume su trabajo, pues suelen asociar el tiempo con el horario cumplido, sin considerar las tareas esenciales al mismo que se realizan en horarios no laborales. Del mismo modo, los esfuerzos cognitivos que insume la resolución de problemas, que no desaparecen al finalizar la jornada, o que se hacen presentes aún fuera del horario de trabajo por los efectos de la comunicación informal, no son contemplados como tiempo de trabajo. Esta situación se refleja en gran parte de los docentes (89.34\%) quienes afirman trabajar sólo cinco días a la semana ${ }^{1}$, entendiendo el tiempo de trabajo según lo consignado en sus Declaraciones Juradas de Cargo y sólo un pequeño grupo parece notar la ampliación velada del tiempo de trabajo al que se los somete. $\mathrm{Al}$ entrecruzar estos datos con los provenientes de otros interrogantes relacionados con esta cuestión, como el tiempo de descanso, se puede advertir que sólo un 33,5\% contesta apegado al tiempo oficial, mientras que los demás reconocen que no descansan las 48 horas semanales que deberían según lo estipulado como tiempo de trabajo remunerado. Lo mismo ocurre con la consulta sobre el trabajo en días no laborales para el sector, donde los dichos se contradicen y muchos docentes reconocen trabajar con diversa carga horaria extra en esos

${ }^{1}$ El sector no trabaja sábados y domingos, salvo circunstancialmente el personal directivo que cuenta en su carga de trabajo las guardias destinadas a emergencias o eventualidades no planificadas en el calendario escolar. 
días. Lo que conduce a sostener que los docentes trabajan más tiempo, sin remuneración, que el consignado en los documentos oficiales.

Siguiendo el esquema de Gollac (citado por Neffa, 2015), se puede entender que los docentes subliman los aspectos asociados al tiempo de trabajo, donde la organización del mismo admite horas laborales no remuneradas, como mecanismo para minimizar el impacto negativo de esta situación sobre su salud mental y emocional, al negar consciente o inconscientemente el tiempo extra de trabajo con los esfuerzos materiales y cognitivos que lo acompañan. Lo que se presenta como un factor de resistencia para que el sector logre percatarse de los RPST que acompañan su trabajo, a la vez que dificulta la difusión, el debate y el posicionamiento de tales Riesgos como temas de interés.

Sin bien las ampliaciones de jornada existen en el mundo del trabajo, vienen acompañadas por pagos extra, que podrían observarse según entiende Siegrist (citado por Neffa, 2015) como recompensa por el aumento en la demanda de trabajo. El análisis de los datos pone en evidencia que el pago por horas suplementarias, no es una categoría que se liquide en Educación, por esta razón, el tiempo insumido para el trabajo que se halla por fuera de lo declarado no es compensado con retribución salarial. Lo mismo ocurre con el tiempo que se destina al trabajo en días no laborales, sábados, domingos y feriados, donde el 95,94\% afirma no haber recibido nunca este tipo de reparación por dedicar su tiempo libre a actividades laborales. Cabe destacar que el Sistema Educativo acepta la discrecionalidad de las Escuelas para otorgar compensatorios en días u horas de trabajo, sin incremento en la remuneración, para cubrir las ampliaciones de jornada para el personal Directivo, administrativo y técnico, no así para los cargos frente a grado que no reciben compensatorio alguno.

Otro tanto ocurre con el tiempo y esfuerzo que se destina a actividades esenciales para la tarea, que según el contenido del trabajo son necesarias para su realización formando parte de su carga y aunque no se conciban desde la organización del trabajo como sujetas a salario, incrementan la carga psíquica, cognitiva y física del trabajador a la que se refiere Gollac (citado por Neffa, 2015), pues se realizan fuera de los días y horarios declarados, generalmente en el domicilio particular del docente e insumiendo parte de su horario de descanso. En este sentido se naturaliza como carga no remunerada la planificación de clases, la realización de informes, la corrección de exámenes, la confección de material didáctico, la organización y elaboración de ornamentación para actos escolares, entre otras actividades. En efecto, cuando los docentes fueron consultados sobre si llevan trabajo para realizar en su domicilio el 89,34\% contestó que lo hacen, de igual manera el 89\% reconoció que sigue pensando en su trabajo cuando ya no está trabajando ${ }^{2}$. Además, una cantidad importante de encuestados manifestaron ser contactados fuera de los horarios de trabajo por motivos laborales, lo cual permite entrever

${ }^{2}$ Aunque el $80 \%$ no logró determinar cuánto de su tiempo particular dedican a esta actividad. 
que el trabajo docente es de tiempo completo aunque sólo se remunera tiempo parcial, el que se realiza en la escuela. Como se trabaja más tiempo del que se declara y se paga, la situación contribuye con la inequidad entre el trabajo y la recompensa de la que habla Siegrist (citado por Neffa, 2015). Entre las cuestiones sin responder y los que contestaron que no trabajan en su domicilio, no se puede inferir necesariamente que lo señalado en la encuesta se deba específicamente a lo que se interroga, puesto que cabría la posibilidad de que los sujetos nieguen inconscientemente esta modalidad de trabajo, como también que no adviertan o naturalicen la presencia de esta modalidad.

La intensidad de la demanda de trabajo abarca la cantidad de trabajo y el tiempo del que se dispone para realizarlo, que también incluye los aspectos como el ritmo y las interrupciones a las que se somete al trabajador. Entonces, aspectos como plazos, metas u objetivos a cumplir, trabajo bajo presión, responder a demandas externas, entre otros elementos que potencian el trabajo, conforman el conjunto de la cantidad de trabajo que se requiere realizar en un período dado, haciendo que la propia organización interfiera con el ritmo de trabajo de su personal. No obstante, los docentes no alcanzan a vislumbrar las mencionadas exigencias como factores que interfieran con sus ritmos de trabajo, pues en su gran mayoría no responden la cuestión, aunque más adelante un 63\% reconoce que existen plazos fijados por la patronal que inciden sobre los ritmos de trabajo, por lo tanto tal contradicción indica desconocimiento o poca comprensión sobre el tema. Incluso cuando se pregunta si su trabajo requiere alcanzar metas predeterminadas el $86 \%$ contesta que sí. Si bien el $74 \%$ responde que le es posible modificar estas metas, no se cree que los docentes se refieran al calendario escolar o a las presentaciones con vencimiento, sino a cierta laxitud contemplada dentro de los plazos establecidos que la institución puede aplicar discrecionalmente, sin que esto implique el control del trabajador sobre la tarea para imprimir su propio ritmo de trabajo, aunque puede percibirse por los mismos de este modo. Lo cual permite deducir que los docentes no alcanzan a alertar el grado de control de la organización sobre los ritmos de su trabajo. Esta falta de conciencia queda expuesta en las respuestas relacionadas con las dificultades para alcanzar metas pre-establecidas, donde el 79\% de los encuestados estiman que tienen dificultades para lograrlas. Lo mismo ocurre con el trabajo bajo presión donde la disparidad entre las respuestas y las ausencias de la misma, muestran la falta de reconocimiento o aceptación sobre el tema. Esto se evidencia también al relacionar los datos, puesto que el 81\% no vivencia que la frecuencia o premura con que debe hacer su trabajo afecta el desempeño e implica una carga material y emocional más elevada, al necesitar realizar con mayor rapidez una operación que requeriría más tiempo y cuidado para hacerla bien.

Para Gollac (citado por Neffa, 2015), factores como consignas contradictorias, incidentes, interrupciones, perturban y retrasan la realización de la tarea, interfiriendo con los ritmos de trabajo, intensificando la carga mental del mismo y los esfuerzos cognitivos empleados en la resolución de situaciones dilemáticas, que ponen en riesgo la salud, física, psíquica y mental del 
trabajador. Según se deriva de los datos los docentes no se percatan de la importancia de este problema, pues respecto a interrumpir la tarea que están desarrollando para realizar actividades imprevistas o someter a examen muchas cosas a la vez, menos de la mitad estima que esto implica aspectos negativos para su trabajo. Lo cual denota que los incidentes, interrupciones y otras situaciones que perturban y retrasan la realización de la tarea, al igual que los esfuerzos cognitivos que demanda el atender varias situaciones al mismo tiempo o pensar diversas cosas en el mismo plazo temporal, no son considerados por los docentes como factores de RPST.

La organización del trabajo en Educación interpreta la educación como un sistema secuenciado donde los contenidos se integran entre sí en núcleos que crecen en complejidad, por ello la actividad de enseñar requiere la articulación entre espacios y niveles, propiciando la dependencia del propio trabajo con el que realizan otros, que como tal afecta los ritmos individuales de trabajo. Esta situación es admitida por los docentes quienes estiman importante la conversación entre colegas y alumnos para desarrollar la tarea, lo que evidencia también que otorgan cierto valor a las relaciones que se producen dentro de la organización. No obstante, cuando se los interroga sobre la dependencia de su trabajo con el que desempeñan sus compañeros las respuestas resultan dispares. Tal situación puede deberse a que dicha interconexión afecta los ritmos del trabajo retrasando la tarea al punto de preferir evitarla, aunque queda abierta la posibilidad de que tal vinculación no se realice porque las relaciones sociales en el trabajo generan un clima que la obstaculiza.

Sobre la ejecución de tareas que demandan acciones repetitivas como sellar documentos, coser expedientes, recibir notas del público, ordenar expedientes, se estima que quienes respondieron esta cuestión son los docentes que ocupan puestos de trabajo en la parte administrativa, que son los expuestos a riesgos como el estrés, que para Karasek (citado por Neffa, 2015) proviene, entre otras cosas, de la resistencia de los trabajadores con cierto nivel de formación a los trabajos repetitivos y monótonos.

En términos generales, se puede colegir que las demandas del trabajo entendidas como "las exigencias psíquicas y mentales sobre: cuánto se trabaja, cantidad o volumen de trabajo realizado, la presión de tiempo, el nivel de atención exigido, la necesidad de hacer frente a incidentes e interrupciones Imprevistas" (Neffa, 2015, p.250), no son vistos por los docentes como factores de RPST.

\subsection{Autonomía y margen de control sobre la actividad}

Siguiendo a Neffa (2015), la autonomía y margen de control del trabajador sobre su actividad, contiene aspectos como el grado de decisión y control del trabajador sobre la tarea, incluyendo la posibilidad de influir sobre sus horarios, vacaciones, licencias y permisos, que al perderse implican Riesgos que se contemplan como factores de RPST. En la formulación de su 
modelo Karasek (citado por Neffa, 2015) alude a la interconexión necesaria entre demandaautonomía-control, como forma de eludir los factores de Riesgo Psicosocial. En este sentido la autonomía consiste en:

La capacidad de decisión para ejecutar las tareas utilizando las calificaciones y competencias propias, decidir cómo hacer las tareas, establecer una secuencia entre ellas, regular las pausas y el ritmo de trabajo y, en última instancia, trabajar según su propio estilo, que es creador de identidad (Neffa, 2015, p. 250).

Dentro de este contexto el control remite a la auto-vigilancia, aspecto esencial de la au-

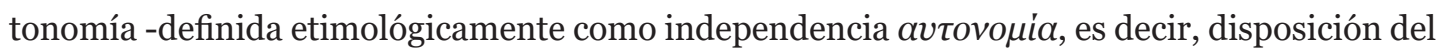

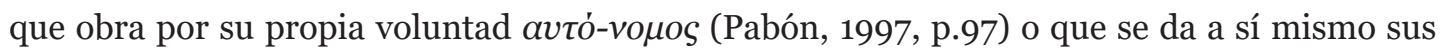
propias reglas-, que atiende a la autorregulación del proceso de trabajo que potencia el desarrollo de habilidades. Por ende, el equilibrio entre demandas organizacionales, grado de control del trabajador sobre su trabajo y autonomía, confluyen en un cierto margen de latitud decisional ${ }^{3}$, donde la persona pone en acción sus competencias profesionales para adaptar las tareas al trabajo prescripto.

En el ejercicio de la profesión docente la expresión 'libertad de cátedra' suele utilizarse como sinónimo de autonomía. No obstante, disponer de la autoridad para seleccionar o recortar ciertos temas a partir de los contenidos mínimos estipulados en el Currículum con el fin de desarrollarlos dentro del aula, poder elegir una estrategia didáctica o implementar una teoría pedagógica, sólo demuestran que el conocimiento sobre el área permite la realización del trabajo. Estas acciones se entienden más como rasgos individuales del trabajo que como signos de libre disposición sobre la tarea, que no aportan indicios suficientes para aseverar la independencia del trabajador para organizar su trabajo de la manera que mejor le conviene. Pues el trabajo docente como cualquier otro, sobre todo dentro de la administración pública, es un trabajo regulado por leyes, estatutos, normativas y resoluciones, que junto al Currículum constituyen el trabajo prescripto. Además, el concepto 'autonomía' como lo entiende Karasek (citado por Neffa, 2015) es mucho más amplio e incorpora dimensiones sobre las cuales el docente no tiene injerencia, aunque crea que sí la tiene. Esta situación queda en evidencia cuando se interroga a los encuestados acerca de las indicaciones dadas por sus superiores, en relación a si les dicen lo que hay que hacer en general, en este aspecto el $71 \%$ no responde y el 20\% considera que la pregunta no corresponde, lo que permite entrever que los docentes sienten que tienen autonomía en la realización de su trabajo. Esta cuestión se confirma al cruzar el ítem con otro donde se busca saber si las órdenes que reciben son precisas, a lo cual más del 90\% responde, dejando en evidencia que todos siguen algún tipo órdenes. Lo mismo ocurre cuando se pregunta si durante el trabajo arregla personalmente situaciones fuera de lo

${ }^{3}$ Autonomía que proviene de la formación y el desarrollo de competencias profesionales. 
común, donde las respuestas muestran una amplia disparidad en la que la mayoría no dispone de la independencia para hacerlo, por ello, frente a eventualidades de este tipo consultan con un superior ${ }^{4}$. Asimismo el escaso margen de decisión sobre la tarea queda manifiesta en la casi inexistente posibilidad del trabajador para definir y organizar su propio horario de trabajo. Donde más del $77 \%$ trabaja con un horario asignado por la institución educativa, aunque en algunos pocos casos los encuestados observaron que en algún momento de su trayectoria en el sector pudieron modificar sus horarios mediante acuerdos internos entre colegas o directivos, sin embargo esta situación se presenta como la excepción y no como la regla. Otro dato que aporta a sostener que la organización controla plenamente los horarios de trabajo de su personal, se refleja en que los docentes conocen los horarios que tendrán, así se trate del día siguiente, de la próxima semana, o del próximo mes, pudiendo interpretarse como horarios habituales, es decir, que son los mismos todos los días.

En lo que hace a la libertad para tomar licencias, el estatuto que regula la actividad en cada Provincia fija las licencias y permisos del personal en sus diferentes niveles y escalafones teniendo en cuenta su situación de revista, mientras que el Ministerio de Educación Provincial se encarga de determinar los períodos de vacaciones por calendario escolar. En este sentido, la organización del trabajo provoca que la antigüedad en el cargo no afecte los tiempos establecidos para vacaciones o licencias, aunque sí restringe la posibilidad de que el actor disponga de estos beneficios sociales cuando mejor le convenga.

En este contexto siguiendo a Karasek (citado por Neffa, 2015), la baja influencia del trabajador en su puesto expresada en el limitado margen de decisión y autonomía que el trabajador tiene sobre el contenido y las condiciones de su trabajo, pueden aumentar los riesgos de enfermedades, puesto que el trabajo prescripto en sí mismo es considerado un factor de Riesgo con impacto negativo sobre la salud. Con lo cual se cree que, por el reducido margen de autonomía y dependiendo de si su trabajo se ejecuta dentro del aula o en la parte administrativa, los docentes podrían ubicarse entre la tercera y la cuarta situación de RPST que identifica Karasek (citado por Neffa, 2015), es decir, intensidad de trabajo fuerte con poca autonomía, que provoca tensiones y efectos negativos en la salud; e intensidad débil sin mucha autonomía, donde el trabajo se vuelve pasivo y rutinario, estimulando la pérdida de interés en la tarea.

\subsection{Recompensa}

La recompensa abarca el aspecto material, moral y simbólico del trabajo, que desde la perspectiva de Siegrist (citado por Neffa, 2015) se mide en relación con la demanda de trabajo.

En lo que hace a la recompensa financiera, el salario del sector docente es uno de los más bajos de la repartición pública. Para 2014 el 43\% ganaba de 5.000 a menos de 10.000 pesos

${ }^{4}$ Si bien algunos admiten solicitar también el auxilio de compañeros de trabajo, no se puede afirmar que tal aproximación se conciba como apoyo social, aunque sí que la comunicación institucional parece preferir los canales extraoficiales. 
por mes y el 41\% de 3.600 a menos de 5.000, por lo cual la mayoría tenía la sensación de estar mal pagado, sobre todo considerando que el sector es uno de los más profesionalizados al restringir el ingreso de personas sin título o certificación oficial. Si bien los montos están desactualizados a la fecha, esto no quita que el sector perciba bajos salarios. Por esta razón, cuando se solicitó a los docentes que expresen cómo se sentían respecto a su salario y en relación con el trabajo que realizan, el $76 \%$ consideró estar mal pago. Sumando que al interrogarlos sobre la preparación que tienen para el puesto que ocupan, 86\% respondió estar bien formado y $11 \%$ estar sobrecalificado.

Respecto a las sensaciones de orgullo por el trabajo bien hecho, utilidad social de su ocupación y el sentido de formar parte de un equipo, los docentes subliman la carencia financiera autoconvenciéndose de la importancia de su actividad -el 70\% considera que nunca se los carga con tareas inútiles-, por ello sólo el $4 \%$ de los entrevistados se siente explotado en su trabajo, mientras que el resto no se percibe de esta manera.

Aunque la teoría del homo economicus conduzca a entender la recompensa en términos pecuniarios, Siegrist (citado por Neffa, 2015) considera que la recompensa no sólo es material sino también moral y simbólica. Dentro de este contexto, el aprecio de los superiores hacia el esfuerzo del trabajador y la estima de los propios compañeros suelen presentarse como situaciones de gratificación, no obstante, para la mitad de los encuestados este tipo de recompensa simbólica no se produce en su lugar de trabajo.

Sobre las posibilidades de ascenso, la estructura organizativa de las escuelas no ofrece, en términos generales, expectativa de ascenso, salvo para el personal titular que quiera hacer carrera en la parte directiva. Por ello, cuando se pregunta sobre si el esfuerzo es proporcional con las posibilidades de ascenso las respuestas son muy dispares y en su mayoría se contestan negativamente o directamente no se responden. Esto permite entrever que el crecimiento personal del trabajador está exento en la relación laboral y como para Siegrist (citado por Neffa, 2015) las expectativas de crecimiento forman parte de las recompensas que se esperan por el trabajo, la falta de las mismas contribuye a reducir el bienestar general del trabajador.

\subsection{Condiciones y medioambiente de trabajo}

En la categoría CyMAT se incorporan aspectos ergonómicos, de higiene, seguridad y salud del trabajador. En la encuesta realizada se hicieron varias preguntas asociadas con este tipo de condiciones, donde la conclusión general conduce a interpretar que en el sector no se tiene conciencia del impacto de las CyMAT sobre la salud y el bienestar. Si bien los docentes reconocen que el trabajo frente a grado obliga a permanecer parado por períodos prolongados y que las tareas administrativas y directivas conllevan la situación inversa, la desproporcionalidad en las respuestas induce a creer que se ignora la magnitud del daño que pueden provocar 
los aspectos físicos y ergonómicos que acompañan los procesos de trabajo. En este sentido, el permanecer en posturas incómodas, trabajar con pantallas de computadoras sin protección, pequeñas o con teclados incómodos, desarrollar las tareas en espacios reducidos, entrar en contacto con materiales peligrosos, estar expuestos a riesgos de accidentes y las condiciones del ambiente, no se perciben como existentes de modo que los factores de Riesgo se naturalizan como formas 'normales' de trabajo, sin advertir que su causa reside en las condiciones del empleo y en el contenido del proceso de trabajo. En relación con la demanda de atención y precisión en el trabajo, se observan similitudes con el caso anterior ya que los docentes no alcanzan a vislumbrar el grado de atención, concentración y esfuerzo cognitivo que demanda la ejecución de su trabajo, por lo cual las respuestas que se consignan en la encuesta arrojan resultados disímiles entre sí. Del mismo modo, cuando se consulta si en su medioambiente de trabajo padece condiciones como suciedad, humedad, corrientes de aire, malos olores, temperaturas ambientales, mal estado de los sanitarios, ausencia de vista al exterior, mala iluminación, inhalación de materias peligrosas y exposición a riesgos de infecciones, la mayoría responde negativamente o no contesta. Situación por la cual se estima que estos resultados se deben a la falta de conocimiento de los factores de Riesgo, que se corrobora con la aseveración del 72\% de los docentes sobre la ausencia de información sobre el tema. Con lo cual se estima que ni el empleador ni los gremios consideran relevante este problema, incluso $72 \%$ afirma que el empleador no difunde instructivos, documentos o carteleras al respecto. Los encuestados también manifestaron no haber sido convocados para discutir estas cuestiones u otras asociadas a reforzar la seguridad en el puesto de trabajo, ni siquiera las vinculadas con tácticas de evacuación de los edificios, por lo cual, gran parte de los encuestados sienten que no se los provee con instrumentos de protección contra los riesgos a los que son expuestos diariamente en su trabajo. Esto evidencia que si bien los docentes no son conscientes de los Riesgos o naturalizan ciertas condiciones de trabajo, el contacto con la noción de Riesgo puede abrir caminos que conduzcan a la implantación del tema dentro del debate institucional.

Respecto a la salud de los trabajadores, a partir del análisis de las encuestas se pudo establecer que gran parte de los docentes pueden enmarcarse dentro de la concepción tradicional de las CyMAT que entiende a la salud como ausencia de enfermedad, sin reparar en los factores de Riesgo como condicionantes de la salud futura del trabajador. Así, el 69\% de los docentes consideran que están sanos simplemente porque no están enfermos hoy. Sin embargo, no observan ciertos aspectos de su trabajo como causales de problemas de salud, como la presión proveniente del trabajo que se realiza cara a cara -aunque el $94 \%$ confirma que su trabajo acepta mayoritariamente esta modalidad-, la tensión o contenido emocional con los cuales el trabajo carga a quien lo realiza, entre otros, coincidiendo con la postura tradicional sobre las CyMAT y una visión restringida de los RPST. Esta posición se mantiene a pesar que cerca del 50\% admite que sufre situaciones de tensión con el público, se encuentra en su trabajo con 
personas que padecen angustia y que para realizar su tarea debe primero calmar a personas angustiadas, nerviosas, preocupadas o coléricas.

\subsection{Inseguridad en el trabajo}

Esta inseguridad ataña al temor causado por el trabajo. Si bien los docentes reconocen que gran parte del tiempo están obligados a esconder sus emociones o evitar expresar libremente sus opiniones, la sensación general de temor no es aceptada explícitamente. Para esto se esbozan dos vías posibles de interpretación, que la misma no sea reconocida como tal, o que el sistema educativo, específicamente el público, ofrezca cierta sensación de seguridad expresada en sus dispositivos de protección contra los directivos, quienes no tienen autoridad para despedir o sancionar con liviandad. Incluso en algunas oportunidades, dependiendo de la gravedad del caso y de la situación de revista del docente, los sumarios administrativos iniciados no llevan al despido sino al reacomodo del personal, lo cual puede propender a minimizar las sensaciones de miedo o temor, sobre todo para el personal que reviste como titular.

En lo atinente a la evaluación de la tarea, la ‘Hoja de Concepto’ puntúa aspectos objetivos como titulación, asistencia, conocimientos y subjetivos como el compromiso con la institución, de allí se cree emana la disparidad en la respuesta a si los que evalúan el trabajo lo conocen bien. Sobre situaciones de maltrato y acoso laboral, no se pudo determinar si las respuestas negativas provienen de la inexistencia de estas situaciones o de la negación de los docentes.

\section{CONCLUSIÓN}

$\mathrm{Al}$ analizar las encuestas realizadas a los docentes de Corrientes, cotejando sus resultados a la luz de las teorías sobre CyMAT y RPST en función de las categorías: demanda psicológica y esfuerzo requerido; autonomía y margen de control sobre la actividad; recompensa; CyMAT; e inseguridad en el trabajo, se pudo evidenciar la presencia de la concepción tradicional de las CyMAT, como el desconocimiento y naturalización de los RPST, que hacen al sector proclive a resistir el enfoque de los riesgos psicosociales en el trabajo.

Respecto a la demanda psicológica y esfuerzo requerido, el incremento en los tiempos no remunerados, ritmos, intensidad y carga cognitiva del trabajo, son naturalizados y sublimados por el sector. La escasa autonomía y margen de control del trabajador sobre su actividad se invisibilizan detrás de la aparente libertad de la tarea, sin considerar que el trabajo docente continúa siendo regulado y que el trabajador no puede organizar su trabajo como mejor le convenga, sino que debe atenerse a las disposiciones y control de la institución, provocando los ex- 
tremos de fuerte intensidad con poca autonomía, aunque en algunos casos de intensidad débil sin mucha autonomía, ambos igualmente dañinos. La recompensa material es insuficiente, lo mismo que la simbólica proveniente del reconocimiento de los pares, aunque estas carencias suelen sublimarse forzando el sentimiento de utilidad social del trabajo. Al no estar presente la concepción renovadora sobre las CyMAT, se pierden de vista los factores de riesgo, ambientales y ergonómicos, lo que impide que se visualice el riesgo. Finalmente, sobre la inseguridad en la situación de trabajo y empleo no pudo determinarse con exactitud si la misma está presente o no con todos sus factores, por lo cual el tema podría ser objeto de otras indagaciones.

Estas características del sector lo ubican en una posición de vulnerabilidad que al no ser reconocida por sus miembros, dificulta la toma de conciencia sobre los factores de Riesgo a los que son expuestos diariamente en su trabajo, al igual que impide el debate libre y responsable con la patronal sobre los cambios que se requieren implementar para mejorar su medioambiente y condiciones de trabajo y reducir los impactos futuros del trabajo sobre la salud del trabajador de la docencia.

\section{REFERENCIAS BIBLIOGRÁFICAS}

Neffa, J. C. (2018). Los riesgos psicosociales en el trabajo: el marco teórico de la investigación. Corrientes: EUDENE.

Neffa, J. C. (2015). Los riesgos psicosociales en el trabajo: contribución a su estudio. Buenos Aires.: CEIL-CONICET.

Pabón, J. M. (1997). Diccionario Griego-Español. 18 ed. Barcelona: Vox.

\section{CURRICULUM VITAE}

\section{Mirian Graciela Arce}

Magister en Gobierno y Economía Política. Especialista en Gestión de las Relaciones del Trabajo. Profesora y Licenciada en Filosofía. Adjunta en la Cátedra Introducción Filosófica a la Economía y Jefe de Trabajos Prácticos en la Cátedra Metodología de las Ciencias Sociales ambas de la Facultad de Ciencias Económicas de la UNNE. Investigadora Categoría V, Dirección Regional de Categorización, Secretaría de Ciencia y Técnica UNNE.

miriangracielaarce@yahoo.com.ar 